\title{
FINITE GROUPS WITH A SELF-GENTRALIZING SUBGROUP OF ORDER 4
}

\author{
WARREN J. WONG ${ }^{1}$ \\ (Received 27 June 1966)
}

The class of finite groups having a subgroup of order 4 which is its own centralizer has been studied by Suzuki [9], Gorenstein and Walter [6], and the present author [11]. The main purpose of this paper is to strengthen Theorem 5 of [11] by using an early result of Zassenhaus [12]. In particular, we find all groups of the class which are core-free, i.e. which have no nontrivial normal subgroup of odd order. As an application, we make a determination of a certain class of primitive permutation groups.

Our proofs are a little longer than strictly necessary, as we have repeated some of the arguments of Gorenstein and Walter rather than rely on their slightly inaccurate proof of their Theorem II [6].

\section{1}

We say that a 2-group is of semi-dihedral type if it has two generators $\alpha, \beta$ with the defining relations

$$
\alpha^{2^{a}}=\beta^{2}=1, \quad \alpha^{\beta}=\alpha^{2^{a-1}-1}, \quad a \geqq 3 .
$$

Also, if $q=r^{2}$, where $r$ is a power of an odd prime number, then, as in [11], we denote by $H(q)$ the group of all transformations of the projective line $G F(q) \cup\{\infty\}$ over $G F(q)$ of the forms

$$
\begin{aligned}
& x \rightarrow \frac{a x+b}{c x+d}, \\
& x \rightarrow \frac{a x^{r}+b}{c x^{r}+d},
\end{aligned}
$$

where $a, b, c, d \in G F(q)$, and $a d-b c$ is a square in (1) and not a square in (2). The 2-Sylow subgroups of $H(q)$ are of semi-dihedral type, and the transformations of form (1) constitute the group $\operatorname{PSL}(2, q)$, a subgroup of index 2 in $H(q)$.

1 Research partially supported by National Science Foundation grant GP-3986 at the University of Notre Dame. 
THEOREM 1. Let $G$ be a finite group with a non-cyclic subgroup $T$ of order 4 which is its own centralizer in $G$. If $K$ is the largest normal subgroup of odd order in $G$, then $G / K$ is isomorphic with PSL $(3,3), M_{11}, G L(2,3), H(q)$, $\operatorname{PGL}(2, q), P S L(2, q)(q$ odd $), A_{7}$, or a 2-group of dihedral or semi-dihedral type.

Proof. Let $S$ be a 2-Sylow subgroup of $G$ containing $T$. Then $S$ is of dihedral or semi-dihedral type [8, Lemma 4]. There is an involution $\tau$ of $T$ lying in the centre of $S$. Let $\mu$ be one of the other involutions of $T$.

First suppose that $S$ is of dihedral type. Then $C_{G}(\tau)$ has a normal 2complement $U[6$, Lemma 8]. Since $\mu$ centralizes $\tau, \mu$ normalizes $U$. Since $T$ is self-centralizing, the automorphism of $U$ induced by $\mu$ has no nontrivial fixed points, so that $U$ is Abelian. Hence $G / K$ is isomorphic with $P S L(2, q), P G L(2, q)$ ( $q$ odd), $A_{7}$, or $S[6$, Theorem I].

Now suppose that $S$ is of semi-dihedral type. We have four possibilities [11, Theorem 2]:

I. $G / K$ is isomorphic with $S$.

II. $G$ has a normal subgroup $H$ of index 2 , such that $H$ has no normal subgroup of index 2 and has 2 -Sylow subgroup of dihedral type.

III. $G$ has a normal subgroup $H$ of index 2, such that $H$ has no normal subgroup of index 2 and has 2 -Sylow subgroup of generalized quaternion type.

IV. $G$ has no normal subgroup of index 2, all involutions of $G$ are conjugate, and the centralizer of any involution is a group of type III.

In case II, $\tau$ lies in $H$ and we see as before that $C_{H}(\tau)$ has a normal Abelian 2-complement, which is also a 2-complement for $C_{G}(\tau)$. Then $G / K$ is isomorphic with $H(q)$ ( $q$ odd) [11, Theorem 3].

In case III, $K$ is the largest normal subgroup of odd order in $H$, and $\tau K$ lies in the centre of $H / K$, by a theorem of Brauer and Suzuki [3, Theorem 2]. Since $T K / K$ is self-centralizing in $G / K$ [6, Lemma 5], $\mu$ induces an automorphism of $H / K$ of order 2 , with exactly one non-trivial fixed point. The structure of $H / K$ and the automorphism induced by $\mu$ are then determined by a result of Zassenhaus [12, Satz 9]; $H / K$ is isomorphic with $S L(2,3)$, and $G / K$ is isomorphic with $G L(2,3)$.

In case IV $C_{G}(\tau)$ is a group of type III, so that, by what has just been proved, $C_{G}(\tau)$ has a normal subgroup $A$ of odd order such that $C_{G}(\tau) / A$ is isomorphic with $G L(2,3)$. By the proof of [11, Theorem 3], $G / K$ is isomorphic with $P S L(3,3)$ or $M_{11}$. This completes the proof of Theorem 1.

As in [11], let $J$ denote the non-split extension of $S L(2,3)$ by a group of order 2 inducing an outer automorphism of $S L(2,3)$.

TheOREM 2. Let $G$ be a finite group with a cyclic subgroup $T$ of order 4 
which is its own centralizer in $G$. If $K$ is the largest normal subgroup of odd order in $G$, then $G / K$ is isomorphic with $S L(2,3), S L(2,5), A_{7}, P S L(2,7)$, PSL(2,9), PGL(2,3), PGL(2,5), H(9), J, a 2-group of semi-dihedral or generalized quaternion type, a dihedral group of order 8 , or a cyclic group of order 4.

Proof. Let $S$ be a 2-Sylow subgroup of $G$ containing $T$. Then $S$ is of one of the following types [9, Prop. 1]:

I. dihedral of order 8 ;

II. semi-dihedral;

III. generalized quaternion;

IV. cyclic of order 4 .

The involution $\tau$ of $T$ lies in the centre of $S$. Let $\mu$ be a generator of $T$.

By Suzuki's results [9], either $G / K$ is isomorphic with $S L(2,3), S L(2,5)$, $A_{7}, P S L(2,7)$ or $P S L(2,9)$, or else $G$ has a normal subgroup $H$ of index 2 which does not contain $\mu$. We may suppose that the latter is true, and further that $G$ does not have a normal 2-complement. In particular, we do not have case IV.

In cases $I$ and II, the 2-Sylow subgroup $S \cap H$ of $H$ is of dihedral type, and we find as in the proof of Theorem 1 that $C_{G}(\tau)$ has an Abelian 2-complement. Hence $G / K$ is isomorphic with $P G L(2, q)$ or $H(q)$ ( $q$ odd) $[6$, Theorem I], $[11$, Theorem 3]. The centralizer in $P G L(2, q)$ of an element of order 4 has order $q \pm 1$, and the centralizer in $H(q)$ of an element of order 4 not in the subgroup $\operatorname{PSL}(2, q)$ has order $2(r-1)$, where $q=r^{2}$. Since $T K / K$ is self-centralizing in $G / K$, it follows that $G / K$ must be isomorphic with $P G L(2,3), P G L(2,5)$, or $H(9)$.

In case III, the 2-Sylow subgroup $S \cap H$ of $H$ is of generalized quaternion type. By the theorem of Brauer and Suzuki [3, Theorem 2], $\tau K$ lies in the centre of $H / K$. Since $T K / K$ is self-centralizing in $G / K, \mu$ induces an automorphism of $H / K$ of order 2, with exactly one non-trivial fixed point. By the result of $Z$ assenhaus [12, Satz 9 ], $H / K$ is isomorphic with $S L(2,3)$, and $G / K$ is isomorphic with $J$. This completes the proof of Theorem 2.

\section{2}

In both the above theorems, the structure of the group $K$ is quite restricted. Since $T$ induces a group of automorphisms of $K$ having no nontrivial fixed points, the derived group of $K$ is nilpotent, by results of Gorenstein and Herstein [5] and Bauman [1]. More can be said about $K$ in special cases. For example, in the situation of Theorem 2, $K$ is Abelian if $G / K$ is isomorphic with $S L(2,3), S L(2,5), P S L(2,7)$ or $\operatorname{PSL}(2,9)$, and $K$ is trivial if $G / K$ is isomorphic with $A_{7}[9]$. The following is another result of this type. 
Theorem 3. In Theorem 1 , if $G / K$ is isomorphic with $M_{11}$ or $A_{7}$, then $K=\{1\}$.

Proof. Suppose that $K$ is non-trivial. Let $L$ be a normal subgroup of $K$ such that $K / L$ is a chief factor of $G$. Since $K$ is solvable, $K / L$ is an elementary Abelian $p$-group for some prime number $p$, and the action of $G / K$ on $K / L$ may be regarded as an irreducible representation of $G / K$ over the field of $p$ elements. The restriction of this representation to the group $T K / K$ does not contain the 1-representation as a constituent, since $T L / L$ is selfcentralizing in $G / L$. This will be true also of any absolutely irreducible constituent of the representation. Hence we obtain an absolutely irreducible Brauer character of $G / K$, the sum of whose values on the elements of $T K / K$ is 0 . Since $M_{11}$ and $A_{7}$ each have only one class of involutions, we have an absolutely irreducible Brauer character $\varphi$ of $M_{11}$ or $A_{7}$ such that

$$
\varphi(1)+3 \varphi(\tau)=0,
$$

where $\tau$ is any involution. Thus it is enough to show that $M_{11}$ and $A_{7}$ do not have such a Brauer character for any odd prime $p$.

The ordinary character tables of $M_{11}$ and $A_{7}$ are known. (For example, they are partly computed in [11] and [9] and can easily be completed by using the orthogonality relations.) The Brauer characters for any odd prime other than 3 can easily be found by using results of Brauer on blocks of defect 1 [2]. In all cases one can check that no Brauer character with property (3) exists. Thus we may assume that $p=\mathbf{3}$.

Since we now have blocks of defect 2 , it is more difficult to find the Brauer characters explicitly. However, $M_{11}$ has a subgroup isomorphic with $P S L(2,11)$, for which the ordinary character table is known [7], and whose Brauer characters $\theta$ for the prime 3 can easily be found. For every $\theta$ we find that $\theta(1)+3 \theta(\tau)>0$, for any involution $\tau$, so that no Brauer character of $M_{11}$ can have the property (3). For $A_{7}$, we have a subgroup isomorphic with the symmetric group $S_{5}$, for which the ordinary character table is well known or easily calculated, and whose Brauer characters for the prime 3 can easily be found. We find that there is no linear combination $\varphi$ of irreducible Brauer characters of $S_{5}$ with positive integer coefficients such that (3) holds for every involution $\tau$ of $S_{5}$. Hence $A_{7}$ has no Brauer character of the required type. This completes the proof of Theorem 3.

As an application of Theorem 1 , we have the following result.

THEOREM 4. Let $G$ be a primitive permutation group such that the subgroup $H$ leaving one letter fixed is isomorphic as an abstract group with the symmetric group $S_{4}$ of degree 4 . Then one of the following holds: 
(i) $G$ has a regular normal elementary Abelian subgroup of order $p^{3}$, $p$ an odd prime number.

(ii) $G$ is isomorphic with $\operatorname{PSL}(\mathbf{2}, q)$, where $q$ is a prime number, $q \equiv \pm \mathbf{1}$ $(\bmod 8)$.

(iii) $G$ is isomorphic with $P G L(2, q)$, where $q$ is a prime number greater than 3 and $q \equiv \pm 3(\bmod 8)$, or $q=3^{t}, t$ an odd prime number.

(iv) $G$ is isomorphic with $\operatorname{PSL}(3,3)$.

Proof. By primitivity of $G, H$ is a maximal subgroup of $G$ containing no non-trivial normal subgroup of $G$. It follows that if $T$ is the normal subgroup of order 4 in $H$ then $H=N_{G}(T)$. Hence $C_{G}(T)=C_{H}(T)=T$, and we have the situation of Theorem 1 .

If $K$ is a non-trivial normal subgroup of odd order in $G$, then $K$ is a transitive subgroup [10, Theorem 8.8]. Since $S_{4}$ has no non-trivial normal subgroup of odd order, $H \cap K=\{1\}$, so that $K$ is regular. Since $K$ is solvable, $K$ is an elementary Abelian $p$-group for some odd prime $p[10$, Theorem 11.5]. The action of $H$ on $K$ may be regarded as a faithful irreducible representation of $H$ over the field of $p$ elements. From the character table of $S_{4}$, we easily see that $H$ has precisely two faithful irreducible representations over any field of odd characteristic, both of degree 3. Hence $K$ has order $p^{3}$, and we have case (i) of the theorem.

If $G$ has no non-trivial normal subgroup of odd order, then Theorem 1 implies that $G$ is isomorphic with $\operatorname{PSL}(3,3), M_{11}, G L(2,3), H(q), P G L(2, q)$, $\operatorname{PSL}(2, q)$, or $A_{7}$.

Since $M_{11}$ has semi-dihedral 2-Sylow subgroups it has only one conjugacy class of non-cyclic subgroups of order 4 , which are all self-centralizing. Hence there can be only one class of subgroups isomorphic to $S_{4}$, viz. the normalizers of these subgroups of order 4 . Since $M_{11}$ has a subgroup isomorphic with $A_{6}$, which in turn has a subgroup isomorphic with $S_{4}$, it follows that $M_{11}$ has no maximal subgroup isomorphic with $S_{4}$.

The only subgroup of $G L(2,3)$ of order 24 is $S L(2,3)$, which is not isomorphic with $S_{4}$.

Since $S_{4}$ is generated by involutions, and all the involutions of $H(q)$ are contained in the normal subgroup $P S L(2, q), H(q)$ cannot have a maximal subgroup isomorphic with $S_{\mathbf{4}}$.

In the permutation representation of $A_{7}$ of degree 7 , a self-centralizing non-cyclic subgroup $T$ of order 4 leaves exactly one letter fixed. Hence the normalizer of $T$ in $A_{7}$ is contained in a subgroup isomorphic with $A_{6}$, and is not a maximal subgroup of $A_{7}$.

Hence $G$ is isomorphic with $\operatorname{PSL}(3,3), P G L(2, q)$ or $P S L(2, q)$ ( $q$ odd). The subgroups of $P S L(2, q)$ and $P G L(2, q)$ are known [4], and it is straightforward to verify that there is a maximal subgroup isomorphic to $S_{4}$ for 
exactly the values of $q$ given in the statement of the theorem. This completes the proof of Theorem 4.

REMARKs. 1. To see that case (iv) of Theorem 4 does occur, consider $\operatorname{PSL}(\mathbf{3}, \mathbf{3})=S L(3,3)$ in its natural representation on a 3 -dimensional vector space over the field $G F(3)$. If $L_{1}, L_{2}, L_{3}$ are three independent onedimensional subspaces, then the elements of $\operatorname{PSL}(3,3)$ which leave $L_{1}$, $L_{2}, L_{3}$ invariant form a non-cyclic subgroup $T$ of order 4 , whose normalizer $H$ consists of all elements of $P S L(3,3)$ which permute $L_{1}, L_{2}, L_{3}$ among themselves. Thus $H$ is isomorphic with $S_{4}$. If $H$ were not maximal in $\operatorname{PSL}(\mathbf{3}, \mathbf{3})$, then $P S L(\mathbf{3}, \mathbf{3})$ would contain a subgroup of one of the types (i), (ii), (iii) of Theorem 4. A comparison of orders shows that this is impossible. Hence $H$ is maximal in $\operatorname{PSL}(3,3)$, and we have case (iv).

2. We saw in the proof of Theorem 4 that there are exactly two nonisomorphic groups of type (i) for each odd prime $p$. In each case there is only one conjugacy class of subgroups isomorphic with $S_{4}$, and so there is essentially only one representation as a permutation group satisfying the hypotheses of Theorem 4. In case (ii) there is essentially only one permutation group for each $q$, since the subgroups of $\operatorname{PSL}(2, q)$ isomorphic to $S_{4}$ are all conjugate in $P G L(2, q)$ [4]. In cases (iii) and (jv) all subgroups isomorphic with $S_{4}$ are conjugate, so that again in each case there is essentially only one representation as a permutation group of the type considered.

Note added in proof. It has come to the author's notice that part of the results of this paper have been obtained by V. D. Mazurov in his article "Finite groups with a given Sylow 2-subgroup", Doklady Akad. Nauk 168 (1966) 519-521, (Soviet Math. 7 (1966), 678-680). His proofs appear rather longer and less elementary than those of the present paper, since he uses the classification by Gorenstein and Walter of all finite groups with dihedral 2-Sylow subgroups, instead of the result of Zassenhaus.

\section{References}

[1] S. Bauman, 'The Klein group as a fixed point free automorphism group', Ph. D. thesis, University of Illinois, 1962.

[2] R. Brauer, 'Investigations on group characters', Annals of Math. 42 (1941), 936-958.

[3] R. Brauer, 'Some applications of the theory of blocks of characters of finite groups II', $J$. of Algebra 1 (1964), 307-334.

[4] L. E. Dickson, Linear groups, (Dover, New York, 1958).

[5] D. Gorenstein and I. N. Herstein, 'Finite groups admitting a fixed-point-free automorphism of order 4', Amer. J. Math. 83 (1961) 71-78.

[6] D. Gorenstein and J. H. Walter, 'On finite groups with dihedral Sylow 2-subgroups', Illinois Jour. Math. 6 (1962), $553-593$.

[7] I. Schur, 'Untersuchungen über die Darstellung der endlichen Gruppen durch gebrochene lineare Substitutionen', Jour. für die reine u. angew. Math. 132 (1907), 85-137. 
[8] M. Suzuki, 'A characterization of simple groups $L F(2, p)$ ', J. Fac. Sci. Univ. Tokyo (Sect. I) 6 (1951), $259-293$.

[9] M. Suzuki, 'On finite groups containing an element of order four which commutes only with its powers', Illinois Jour. Math. 3 (1959), 255-271.

[10] H. Wielandt, Finite Permutation Groups, (Academic Press, New York, 1964).

[11] W. J. Wong, 'On finite groups whose 2-Sylow subgroups have cyclic subgroups of index 2', J. Australian Math. Soc. 4 (1964), 90-112.

[12] H. Zassenhaus, 'Kennzeichnung endlicher linearer Gruppen als Permutationsgruppen', Abh. math. Sem. Univ. Hamburg 11 (1936), 17-40.

\section{University of Notre Dame}

Notre Dame, Indiana 